\title{
Sound Shell Model for Acoustic Gravitational Wave Production at a First-Order Phase Transition in the Early Universe
}

\section{Hindmarsh, Mark}

2018-02-13

Hindmarsh , M 2018 , ' Sound Shell Model for Acoustic Gravitational Wave Production at a First-Order Phase Transition in the Early Universe ', Physical Review Letters , vol. 120 , no. 7,071301 . https://doi.org/10.1103/PhysRevLett.120.071301

http://hdl.handle.net/10138/233184

https://doi.org/10.1103/PhysRevLett.120.071301

cc_by

publishedVersion

Downloaded from Helda, University of Helsinki institutional repository.

This is an electronic reprint of the original article.

This reprint may differ from the original in pagination and typographic detail.

Please cite the original version. 


\title{
Sound Shell Model for Acoustic Gravitational Wave Production at a First-Order Phase Transition in the Early Universe
}

\author{
Mark Hindmarsh* \\ Department of Physics and Astronomy, University of Sussex, Falmer, Brighton BN1 9QH, United Kingdom \\ and Department of Physics and Helsinki Institute of Physics, PL 64, FI-00014 University of Helsinki, Finland
}

(Received 4 October 2016; revised manuscript received 18 December 2017; published 13 February 2018)

\begin{abstract}
A model for the acoustic production of gravitational waves at a first-order phase transition is presented. The source of gravitational radiation is the sound waves generated by the explosive growth of bubbles of the stable phase. The model assumes that the sound waves are linear and that their power spectrum is determined by the characteristic form of the sound shell around the expanding bubble. The predicted power spectrum has two length scales, the average bubble separation and the sound shell width when the bubbles collide. The peak of the power spectrum is at wave numbers set by the sound shell width. For a higher wave number $k$, the power spectrum decreases to $k^{-3}$. At wave numbers below the inverse bubble separation, the power spectrum goes to $k^{5}$. For bubble wall speeds near the speed of sound where these two length scales are distinguished, there is an intermediate $k^{1}$ power law. The detailed dependence of the power spectrum on the wall speed and the other parameters of the phase transition raises the possibility of their constraint or measurement at a future space-based gravitational wave observatory such as LISA.
\end{abstract}

DOI: 10.1103/PhysRevLett.120.071301

Interest in gravitational waves from a first-order electroweak phase transition in the early Universe [1-3] has greatly increased following the European Space Agency's approval of a space-based gravitational wave observatory [4] and the detection of gravitational waves from a merging black hole binary [5]. At the same time, it has been realized that early work on gravitational waves from a thermal phase transition $[6,7]$ greatly underestimated their energy density [8]. The first three-dimensional hydrodynamic simulations $[8,9]$ revealed that the dominant source of gravitational waves was acoustic production from sound waves generated by the explosive growth of bubbles of the stable phase. In fact, it had been pointed out long before that sound waves were a source of gravitational waves [10], but subsequent work had not appreciated that the sound wave source persisted for long after the phase transition completed, hence boosting the signal by orders of magnitude. The original model of gravitational radiation from the colliding bubble walls may still be relevant for nearvacuum transitions [11], and a semianalytic approach has recently been developed [12].

The simulations in Refs. $[8,9]$ revealed a power spectrum peaked at a wavelength around the average bubble separation $R_{*}$, with a power law $k^{-p}$ at wave number $k \gg R_{*}^{-1}$. Where the power law was clear, the index was somewhere in the range $-3 \lesssim p \lesssim-4$. There was also evidence for some structure in the peak: where the bubble wall speed $v_{w}$ was closer to the speed of sound, the peak was broader. Understanding the gravitational wave power spectrum is of great importance for LISA's detection prospects [13], so it is vital to have a better physical understanding of the numerical simulations.
In this Letter, I outline a model for the acoustic gravitational wave power spectrum based on the observation that the shells of compression and rarefaction (i.e., sound pulses) around the expanding bubble of the stable phase continue to propagate after the phase boundaries driving them have disappeared. The radial velocity field $v(r, t)$ surrounding the bubble takes a characteristic invariant profile $v_{\text {ip }}(\xi)$, with $\xi=r / t$, which acts as the initial condition for the sound wave at the bubble wall collision time $t_{\mathrm{bc}}$. The subsequent local fluid velocity is the superposition of the sound waves from many sound shells, and it can be treated as a Gaussian random field while linearity is maintained. The power spectrum of the velocity field is computable from the velocity profile $v_{\text {ip }}(\xi)$, which has a simple form in linearized hydrodynamics. The gravitational wave power spectrum can then be computed from a Gaussian velocity field by a simple convolution of the power spectrum $[14,15]$. The model is distinguished from earlier modeling of the velocity field and shear stress correlations $[16,17]$ by the recognition that long-lasting sound waves are the main source of gravitational radiation, and by the computation of their power spectrum from the hydrodynamic solution.

In the sound shell model the gravitational wave power spectrum has a characteristic double broken power law form (39) with two length scales, the bubble separation $R_{*}$ and the sound shell thickness $\Delta R_{*}$. For wall speeds near the speed of sound $c_{s}$, the sound shell is thin, and there is a characteristic $k^{1}$ power law in the range $R_{*}^{-1} \lesssim k \lesssim \Delta R_{*}^{-1}$. For large $k \Delta R_{*}$ values, the power law index is -3 , and for small $k R_{*}$ values, it is +5 . 
In the following I recap how the gravitational wave power spectrum can be derived from the shear stress unequal time correlator (UETC), and how the shear stress power spectrum is found from the velocity power spectrum. I then present the model for the velocity power spectrum, derived from an incoherent superposition of the power spectra from randomly placed sound shells, which are launched into free propagation on the collision of the phase boundaries.

In its simplest form, the model assumes that all fluid velocities are nonrelativistic, and that the bubble separation is much less than the Hubble distance at the transition $1 / H_{*}$. A corollary is that the duration of the transition is much shorter than the Hubble time. We find it convenient to distinguish between the spectral density of a field with Fourier coefficients $f_{\mathbf{k}}, P_{f}=\left|f_{\mathbf{k}}\right|^{2}$ and the power spectrum $\mathcal{P}_{f}=k^{3}\left|f_{\mathbf{k}}\right|^{2} / 2 \pi^{2}$.

We start by defining

$$
\tau^{i j}=\Gamma \epsilon v^{i} v^{j},
$$

where $v^{i}$ is the fluid velocity, $\epsilon$ is the energy density, and $\Gamma$ is the adiabatic index of the fluid (equal to $4 / 3$ for an ideal relativistic gas). This is the relevant part of the energymomentum tensor for gravitational wave production from a fluid moving nonrelativistically.

The fluid shear stress UETC $\Pi^{2}$ is then defined using

$\lambda_{i j, k l}\left(\mathbf{k}_{1}\right)\left\langle\tau_{\mathbf{k}_{1}}^{i j}\left(t_{1}\right) \tau_{\mathbf{k}_{2}}^{k l}\left(t_{2}\right)\right\rangle=\Pi^{2}\left(k_{1}, t_{1}, t_{2}\right)(2 \pi)^{3} \delta\left(\mathbf{k}_{1}+\mathbf{k}_{2}\right)$,

where $\lambda_{i j, k l}(\mathbf{k})$ is the transverse-traceless projector for symmetric tensors. Assuming that the fluid shear stress fluctuations have a characteristic length scale $L_{f}$ and are stationary well after they are created, we may write

$$
\Pi^{2}\left(k, t_{1}, t_{2}\right) \simeq\left(\Gamma \bar{\epsilon} \bar{U}_{f}^{2}\right)^{2} L_{f}^{3} \tilde{\Pi}^{2}\left(k L_{f}, \zeta\right),
$$

where $\zeta=k\left(t_{1}-t_{2}\right), \bar{\epsilon}$ is the mean energy density, and $\bar{U}_{f}^{2}$ is the mean square fluid velocity (weighted by the energy density). We assume that the energy density fluctuations are of order $\bar{U}_{f}$ and can be neglected. We will justify the assumption of stationarity below.

The gravitational wave $(\mathrm{gw})$ power spectrum $\mathcal{P}_{\mathrm{gw}}(k)=$ $d \Omega_{\mathrm{gw}}(k) / d \ln (k)$ of an acoustic source with lifetime $\tau_{v}$ and a length scale $L_{f}$ operating when the Hubble rate is $H_{*}$ can be shown to be [9]

$$
\mathcal{P}_{\mathrm{gw}}(k)=3 \Gamma^{2} \bar{U}_{f}^{4}\left(H_{*} \tau_{v}\right)\left(H_{*} L_{f}\right) \frac{\left(k L_{f}\right)^{3}}{2 \pi^{2}} \tilde{P}_{\mathrm{gw}}\left(k L_{f}\right) .
$$

The dimensionless spectral density $\tilde{P}_{\mathrm{gw}}\left(k L_{f}\right)$ is found from integrating the scaled fluid shear stress UETC with the appropriate Green's functions,

$$
\tilde{P}_{\mathrm{gw}}\left(k L_{f}\right)=\frac{1}{k L_{f}} \int d \zeta \frac{\cos (\zeta)}{2} \tilde{\Pi}^{2}\left(k L_{f}, \zeta\right)
$$

It was shown in Ref. [9] that, in an expanding universe, times and distances are to be interpreted as conformal and comoving in Eq. (4). It was also shown that viscous damping is negligible for the scales of interest, and that, provided the correlation scale $L_{f}$ is much less than the Hubble distance, the effective source lifetime $\tau_{v}$ is precisely the Hubble time $H_{*}^{-1}$ [9]. Dissipation can also arise through the formation of shocks (and eventually turbulence), after a time $[18,19]$

$$
\tau_{\mathrm{sh}} \sim L_{f} / \bar{U}_{f}
$$

We will assume that $\bar{U}_{f} \ll L_{f} H_{*}$, so turbulence takes more than the Hubble time to develop, and that the signal is suppressed by a factor $\left(H_{*} \tau_{\mathrm{sh}}\right)^{-1}[9,20]$.

We now assume that the shear stress UETC is generated from a Gaussian random irrotational velocity field $v^{i}(\mathbf{x}, t)$, as is appropriate for sound waves. We denote the Fourier transform

$$
\tilde{v}_{\mathbf{q}}^{i}(t)=\int d^{3} x v^{i}(\mathbf{x}, t) e^{-i \mathbf{q} \cdot \mathbf{x}}
$$

and the longitudinal part of its UETC $G\left(q, t_{1}, t_{2}\right)$ by

$$
\hat{q}^{i} \hat{q}^{j}\left\langle\tilde{v}_{\mathbf{q}_{1}}^{i}\left(t_{1}\right) \tilde{v}_{\mathbf{q}_{2}}^{* j}\left(t_{2}\right)\right\rangle=G\left(q, t_{1}, t_{2}\right)(2 \pi)^{3} \delta\left(\mathbf{q}_{1}-\mathbf{q}_{2}\right) .
$$

It can then be shown that [14-16]

$$
\begin{aligned}
\Pi^{2}\left(k ; t_{1}, t_{2}\right)= & \left(\frac{4}{3} \bar{\epsilon}\right)^{2} \int \frac{d^{3} q}{(2 \pi)^{3}} \frac{q^{2}}{\tilde{q}^{2}}\left(1-\mu^{2}\right)^{2} \\
& \times G\left(q, t_{1}, t_{2}\right) G\left(\tilde{q}, t_{1}, t_{2}\right),
\end{aligned}
$$

where $\tilde{\mathbf{q}}=\mathbf{q}-\mathbf{k}$ and $\mu=\hat{\mathbf{q}} \cdot \hat{\mathbf{k}}$.

Our model velocity field is the superposition of velocity fields from bubbles nucleated at random times $t^{(n)}$ and positions $\mathbf{x}^{(n)}$,

$$
v_{i}(\mathbf{x}, t)=\sum_{n} v_{i}^{(n)}(\mathbf{x}, t) .
$$

The velocity field of the bubbles is taken to be the asymptotic invariant profile, which is radial. The shear stress tensor is initially the sum of nonoverlapping spherically symmetric distributions, and it therefore sources no gravitational waves. The spherical symmetry is lost as soon as the bubble velocity fields start to overlap. Overlaps are assumed to be linear superpositions, justified for low fluid velocities.

When a segment of a bubble wall collides at $t_{\mathrm{bc}}$, removing the local forcing of the fluid, the fluid is launched into free propagation, so the invariant profile is the initial 
condition for the subsequent linear evolution of the velocity field. This acoustic phase [9] lasts much longer than the collision phase and therefore is the dominant source of gravitational radiation, unless very large fluid speeds $\left(\bar{U}_{f} \sim v_{w}\right)$ are generated.

The velocity field in a sound wave obeys the equation

$$
\left(\partial_{t}^{2}-c_{s}^{2} \nabla^{2}\right) v^{i}(\mathbf{x}, t)=0,
$$

whose general solution has the plane wave decomposition

$$
v^{i}(\mathbf{x}, t)=\int \frac{d^{3} q}{(2 \pi)^{3}}\left(v_{\mathbf{q}}^{i} e^{-i \omega t+i \mathbf{q} \cdot \mathbf{x}}+v_{\mathbf{q}}^{* i} e^{i \omega t-i \mathbf{q} \cdot \mathbf{x}}\right),
$$

where $\omega=c_{s} q$. Note the distinction between the plane wave amplitudes $v_{\mathbf{q}}^{i}$ and the Fourier transform of the velocity field $\tilde{v}_{\mathbf{q}}^{i}(t)$.

Writing $\dot{\tilde{v}}_{i}(\mathbf{q}, t)$ for the acceleration field, we see that at the matching time $t_{\mathrm{bc}}$, the plane wave amplitude is

$$
v_{\mathbf{q}}^{i}=\frac{1}{2}\left(\tilde{v}_{\mathbf{q}}^{i}\left(t_{\mathrm{bc}}\right)+\frac{i}{\omega} \dot{\tilde{v}}_{\mathbf{q}}^{i}\left(t_{\mathrm{bc}}\right)\right) e^{i \omega t_{\mathrm{bc}}}
$$

The fields $v^{i}(\mathbf{x}, t)$ and $i^{i}(\mathbf{x}, t)$ are related by the fluid velocity around the colliding bubbles, so the plane wave coefficients $v_{\mathbf{q}_{1}}^{i}$ and $v_{\mathbf{q}_{2}}^{* j}$ are not independent. We will see (26) that the relationship is

$$
v_{\mathbf{q}, i}^{(n)} \simeq-e^{2 i \omega t_{\mathrm{bc}}+i \theta(z)} v_{-\mathbf{q}, i}^{(n) *},
$$

where $\theta$ is a $q$-dependent phase. With this is mind, one can show that

$$
G\left(q, t_{1}, t_{2}\right)=2 P_{v}\left(q_{1}\right) D\left(\omega_{1}, t_{1}, t_{2}\right),
$$

where $P_{v}$ is the spectral density of the plane wave amplitudes, defined using

$$
\left\langle v_{\mathbf{q}_{1}}^{i} v_{\mathbf{q}_{2}}^{* i}\right\rangle=P_{v}\left(q_{1}\right)(2 \pi)^{3} \delta\left(\mathbf{q}_{1}-\mathbf{q}_{2}\right),
$$

and where

$D\left(\omega_{1}, t_{1}, t_{2}\right)=\cos \left[\omega_{1}\left(t_{1}-t_{2}\right)\right]-\cos \left[\omega_{1}\left(t_{1}+t_{2}-2 t_{i}-\theta\right)\right]$.

In the sound shell model, the velocity UETC is the incoherent sum of contributions from collisions at a distribution of times $t_{\mathrm{bc}}$, centered at a time $t_{\mathrm{pc}}$, with a width $\beta^{-1}$. In the acoustic phase, $\beta\left|t_{1}+t_{2}-2 t_{\mathrm{pc}}\right| \gg 1$. For sound waves with frequencies much greater than $\beta$, the second term in Eq. (17) will be suppressed by the integral over collision times. For lower frequencies $\left(c_{s} q / \beta \ll 1\right.$,), the second term is suppressed in the shear stress UETC (9) at late times, as it is oscillatory in the integration variable $q$ for $c_{s} q \gg\left|t_{1}+t_{2}-t_{\mathrm{pc}}\right|^{-1}$.
Hence, the relevant part of the velocity UETC is

$$
G\left(q, t_{1}, t_{2}\right) \simeq 2 P_{v}(q) \cos \left[c_{s} q\left(t_{1}-t_{2}\right)\right],
$$

justifying the stationary assumption in Eq. (3).

With this form of the velocity UETC, the cosines combine to produce delta functions $\delta(k \pm \omega \pm \tilde{\omega})$ in the integral over $t_{1}-t_{2}$ in Eq. (5). Only $k-\omega-\tilde{\omega}$ can vanish, and it follows that

$$
\begin{aligned}
\tilde{P}_{\mathrm{gw}}(y)= & \frac{1}{4 \pi y c_{s}}\left(\frac{1-c_{s}^{2}}{c_{s}^{2}}\right)^{2} \int_{z_{-}}^{z_{+}} \frac{d z}{z} \frac{\left(z-z_{+}\right)^{2}\left(z-z_{-}\right)^{2}}{\left(z_{+}+z_{-}-z\right)} \\
& \times \bar{P}_{v}(z) \bar{P}_{v}\left(z_{+}+z_{-}-z\right),
\end{aligned}
$$

where $y=k L_{f}, z=q L_{f}$, and $z_{ \pm}=\left(y / c_{s}\right)\left[\left(1 \pm c_{s} / 2\right)\right]$.

It is clear that if the velocity power spectrum $\mathcal{P}_{v}$ is $\left(q L_{f}\right)^{n}$ over a range of wave numbers, the gravitational wave power spectrum becomes

$$
\mathcal{P}_{\mathrm{gW}}(k) \sim\left(k L_{f}\right)^{2 n-1} .
$$

We now calculate the spectral density of the plane wave coefficients for the sound shell around a single bubble, nucleated at time $t^{(n)}$ at position $\mathbf{x}^{(n)}$. The velocity field is radial and self-similar, so we can write

$$
v_{i}^{(n)}(\mathbf{x}, t)=\hat{R}_{i}^{(n)} v_{\text {ip }}(\xi),
$$

where $R_{i}^{(n)}=x_{i}-x_{i}^{(n)}, T^{(n)}=t-t^{(n)}$, and $\xi=R^{(n)} / T^{(n)}$. The function $v_{\text {ip }}$ for various wall speeds can be seen in Fig. 3 of Ref. [21]. The width of the velocity profile is determined by $\Delta v_{w}=v_{w}-c_{s}$.

The Fourier transform of the velocity field is

$$
\tilde{v}_{i}^{(n)}(\mathbf{q}, t)=e^{-i \mathbf{q} \cdot \mathbf{x}^{(n)}}\left(T^{(n)}\right)^{3} i \hat{z}^{i} f^{\prime}(z),
$$

where $z^{i}=q^{i} T^{(n)}$ and

$$
f(z)=\frac{4 \pi}{z} \int_{0}^{\infty} d \xi v_{\text {ip }}(\xi) \sin (z \xi) .
$$

The time derivative of $\tilde{v}^{i}$ follows from the time dependence of the invariant profile, so

$$
\dot{\tilde{v}}_{i}^{(n)}(\mathbf{q}, t)=e^{-i \mathbf{q} \cdot \mathbf{x}^{(n)}}\left(T^{(n)}\right)^{2} i \hat{z}_{i} g^{\prime}(z),
$$

where

$$
g(z)=\frac{1}{z} \frac{d}{d z}\left[z^{2} f(z)\right]
$$

The contribution from the $n$th bubble to the coefficient in the plane wave expansion of the general solution is therefore 


$$
v_{\mathbf{q}, i}^{(n)}=i\left(T_{\mathrm{bc}}^{(n)}\right)^{3} \hat{z}_{i} e^{i \omega t_{\mathrm{bc}}-i \mathbf{q} \cdot \mathbf{x}^{(n)}} A(z),
$$

where $T_{\mathrm{bc}}^{(n)}=t_{\mathrm{bc}}-t^{(n)}$ is the bubble lifetime and

$$
A(z)=\frac{1}{2}\left(f^{\prime}(z)+\frac{i}{c_{s} z} g^{\prime}(z)\right) .
$$

Note that now, $z=q T_{\mathrm{bc}}^{(n)}$.

We average over bubble centers and the bubble lifetime distribution $n\left(T_{\mathrm{bc}}\right)$, which is calculable from the bubble nucleation rate $[22,23]$. On dimensional grounds,

$$
n\left(T_{\mathrm{bc}}\right) d T_{\mathrm{bc}}=\frac{\beta}{R_{*}^{3}} \nu\left(\beta T_{\mathrm{bc}}\right) d T_{\mathrm{bc}},
$$

where $\beta=(8 \pi)^{1 / 3} v_{w} / R_{*}[23]$ is the phase transition rate parameter. By definition, $\int n\left(T_{\mathrm{bc}}\right) d T_{\mathrm{bc}}=1 / R_{*}^{3}$, the bubble number density. Hence, the velocity spectral density is

$$
P_{v}(q)=\frac{R_{*}^{3}}{(8 \pi)^{2} v_{w}^{6}} \int d \tilde{T} \nu(\tilde{T}) \tilde{T}^{6}|A(\tilde{T} q / \beta)|^{2},
$$

where $\tilde{T}=\beta T_{\mathrm{bc}}$. The bubble lifetimes peak at around $\tilde{T} \simeq$ $\beta R_{*} / v_{w}$ with similar widths, so if there is a power law $z^{n-3}$ in the one-bubble spectral density $\left|A^{2}\right|$, we have

$$
P_{v}(q) \sim R_{*}^{3}\left(q R_{*}\right)^{n-3} .
$$

Oscillatory features at high $q R_{*}$ values will be averaged over.

The linearized fluid equations have the solution [24,25]

$$
v_{\text {ip }}(\xi)=v_{\max } \frac{v_{w}^{2}}{\xi^{2}} \frac{c_{s}^{2}-\xi^{2}}{c_{s}^{2}-v_{w}^{2}},
$$

where $v_{\max }\left(v_{w}, \alpha\right)$ is computable from the wall speed and the scalar potential difference relative to the total energy density $\alpha[1,18,26]$. For small $\alpha$ values,

$$
v_{\max } \simeq\left\{\begin{array}{ll}
3 \alpha v_{w}\left|\gamma_{w s}^{2}\right|, & \left|\Delta_{w}\right|>O(\sqrt{\alpha}) \\
\sqrt{3 \alpha / 2}, & \left|\Delta_{w}\right|<O(\sqrt{\alpha})
\end{array},\right.
$$

where $\Delta_{w}=\Delta v_{w} / v_{w}$, and $\gamma_{w s}^{2}=c_{s}^{2} /\left(c_{s}^{2}-v_{w}^{2}\right)$. At low fluid speeds, solution (31) is valid for $\xi$ values between $v_{w}$ and $c_{s}$.

Transform (23) can be calculated exactly, giving

$$
\begin{aligned}
f^{\prime}(z)= & -4 \pi v_{\max } v_{w}^{2} c_{s} \frac{1}{c_{s} z}\left(\operatorname{sinc}\left(v_{w} z\right)\right. \\
& \left.+\frac{2 \gamma_{w s}^{2}}{\left(c_{s} z\right)^{2}}\left[\cos \left(c_{s} z\right)-\cos \left(v_{w} z\right)\right]\right), \\
g^{\prime}(z)= & 4 \pi v_{\max } v_{w}^{2} c_{s} \frac{1}{c_{s} z}\left(v_{w} z j_{1}\left(v_{w} z\right)\right. \\
& \left.+2 \gamma_{w s}^{2}\left[\operatorname{sinc}\left(c_{s} z\right)-\operatorname{sinc}\left(v_{w} z\right)\right]\right) .
\end{aligned}
$$

The limits of the power spectrum can now be extracted, distinguishing between the scales $v_{w} z$ and $\Delta v_{w} z$. First, when both $z v_{w}$ and $z \Delta v_{w}$ are small,

$$
|A(z)|^{2} \simeq \frac{4 \pi^{2}}{9} v_{\max }^{2} v_{w}^{6}\left(1+\frac{v_{w}}{c_{s}}\right)^{2} v_{\max }^{2} \Delta_{w}^{2},
$$

with a white noise power spectrum, as expected. For a thin sound shell, we can investigate the range $z \Delta v_{w} \ll 1 \ll z v_{w}$, for which

$$
|A(z)|^{2} \simeq \pi^{2} v_{\max }^{2} v_{w}^{4} c_{s}^{2} \Delta_{w}^{2}\left(c_{s} z\right)^{-2} .
$$

Finally, when both $z v_{w}$ and $z \Delta_{w}$ are large,

$$
|A(z)|^{2} \simeq 4 \pi^{2} v_{\max }^{2} v_{w}^{2} c_{s}^{4}\left(z c_{s}\right)^{-4} .
$$

Hence, the velocity power spectrum becomes

$$
\mathcal{P}_{v}(q) \sim \begin{cases}v_{\max }^{2} \Delta_{w}^{2}\left(q R_{*}\right)^{3}, & q \Delta R_{*}, q R_{*} \ll 1 \\ v_{\max }^{2} \Delta_{w}^{2}\left(q R_{*}\right)^{1}, & q \Delta R_{*} \ll 1 \ll q R_{*}, \\ v_{\max }^{2} \Delta_{w}^{2}\left(q R_{*}\right)^{-1}, & 1 \ll q \Delta R_{*}, R_{*}\end{cases}
$$

where $\Delta R_{*} \simeq \Delta v_{w} / \beta$. The $q^{-1}$ form at large $q$ is a consequence of the compact support of the velocity field for $\xi$ values between $v_{w}$ and $c_{s}$. If the discontinuity at $\xi=v_{w}$ is smoothed over a length scale $\ell$, the power law form no longer applies for $q \gg \ell^{-1}$.

The gravitational wave power spectrum becomes

$$
\frac{\mathcal{P}_{\mathrm{gw}}(k)}{H_{*} R_{*}} \sim \begin{cases}v_{\max }^{4} \Delta_{w}^{4}\left(k R_{*}\right)^{5}, & k \Delta R_{*}, k R_{*} \ll 1 \\ v_{\max }^{4} \Delta_{w}^{4}\left(k R_{*}\right)^{1}, & k \Delta R_{*} \ll 1 \ll k R_{*} . \\ v_{\max }^{4} \Delta_{w}^{4}\left(k R_{*}\right)^{-3}, & 1 \ll k \Delta R_{*}, R_{*}\end{cases}
$$

Form (39) is the main result. Note that the wave number at the peak of the power spectrum is set by the sound shell thickness, not the bubble separation. The gravitational wave power at the peak is $\mathcal{P}_{\mathrm{gw}}^{\text {peak }} \sim v_{\max }^{4}\left|\Delta_{w}\right|^{3}\left(H_{*} R_{*}\right)$. The peak power increases to $\alpha^{4}\left|\Delta_{w}\right|^{-1}\left(H_{*} R_{*}\right)$, except for $\left|\Delta_{w}\right| \lesssim \sqrt{\alpha}$, where it is $\mathcal{P}_{\mathrm{gw}}^{\text {peak }} \sim \alpha^{7 / 2}\left(H_{*} R_{*}\right)$.

Note also that the low wave number power law is steeper than the expected $k^{3}$ [17]. The $k^{5}$ power law can be understood as follows. For causal velocity fields with white noise spectral densities, the shear stress UETC (9) becomes $k^{3}$ at low wave number. The decoherence time of the UETC is $k^{-1}$, so when it is integrated with the Green's function (5), the gravitational wave spectral density becomes $k^{2}$, and the power spectrum $k^{5}$. The widely quoted result [17] that a causally produced gravitational wave power spectrum should go to $k^{3}$ follows from assuming that the shear stress UETC becomes $k^{0}$ at low $k$, and that the decoherence time is independent of $k$. The $k^{5}$ behavior is consistent with causality, which only bounds the power spectrum to be steeper than $k^{3}$. 
At very low $k$, we expect the contribution from bubble wall collisions, which does go to $k^{3}$ [27], to take over. Similarly, for a high wave number, the bubble wall collision signal goes to $k^{-1}[7,11,12]$ and eventually reemerges.

The power law predictions of the sound shell model have recently been compared against results from numerical simulations [20], with some success, particularly for the intermediate and high $k$ power laws. The peak amplitude also scales as predicted, increasing by a factor $O\left(10^{4}\right)$ when $\alpha$ is increased by a factor $O(10)$. It is also shown in Ref. [20] that, for transitions with $H_{*} R_{*} \sim 10^{-2}$, where LISA is most sensitive, a rms fluid velocity of $\bar{U}_{f} \gtrsim 0.05$ gives a signal-to-noise ratio greater than 20 and therefore should be observable. Given that $\bar{U}_{f} \sim v_{\max }$, one can estimate that this corresponds to $\alpha \gtrsim 0.05$ for generic wall speeds $\left|\Delta_{w}\right| \sim 1$.

In summary, a model for the acoustic production of gravitational waves at a first-order phase transition has been outlined. The gravitational wave power spectrum is a double broken power law (39) built from the two physical scales: the bubble separation and the sound shell thickness. For a generic wall speed, these scales are approximately the same, and the form of the power spectrum for both deflagrations and detonations is similar (but not identical). Wall speeds near the speed of sound are distinguished by an intermediate $k^{1}$ power law between the two scales.

The detailed power spectrum depends computably on the wall speed, the bubble separation, the Hubble parameter at the transition, and the latent heat. Future work will exhibit this dependence and explore prospects for the measurement or constraint of the phase transition parameters by accurate determination of a stochastic gravitational wave background.

I am grateful to Nicola Hopkins for her collaboration on early stages of this work, and to Stephan Huber, Kari Rummukainen, and David Weir for many discussions. I have also benefited from exchanges with Chiara Caprini, Germano Nardini, and other members of the eLISA Cosmology Working Group. I acknowledge support from the Science and Technology Facilities Council (Grant No. ST/J000477/1). *m.b.hindmarsh@sussex.ac.uk

[1] P. J. Steinhardt, Phys. Rev. D 25, 2074 (1982).

[2] E. Witten, Phys. Rev. D 30, 272 (1984).

[3] C. Hogan, Phys. Lett. 133B, 172 (1983).

[4] P. A. Seoane et al. (eLISA Consortium), arXiv:1305.5720.

[5] B. P. Abbott et al. (Virgo and LIGO Scientific Collaborations), Phys. Rev. Lett. 116, 061102 (2016).

[6] A. Kosowsky, M. S. Turner, and R. Watkins, Phys. Rev. Lett. 69, 2026 (1992).

[7] S. J. Huber and T. Konstandin, J. Cosmol. Astropart. Phys. 09 (2008) 022.

[8] M. Hindmarsh, S. J. Huber, K. Rummukainen, and D. J. Weir, Phys. Rev. Lett. 112, 041301 (2014).

[9] M. Hindmarsh, S. J. Huber, K. Rummukainen, and D. J. Weir, Phys. Rev. D 92, 123009 (2015).

[10] C. J. Hogan, Mon. Not. R. Astron. Soc. 218, 629 (1986).

[11] D. J. Weir, Phys. Rev. D 93, 124037 (2016).

[12] R. Jinno and M. Takimoto, Phys. Rev. D 95, 024009 (2017)

[13] C. Caprini et al., J. Cosmol. Astropart. Phys. 04 (2016) 001.

[14] A. Kosowsky, A. Mack, and T. Kahniashvili, Phys. Rev. D 66, 024030 (2002).

[15] C. Caprini and R. Durrer, Phys. Rev. D 74, 063521 (2006).

[16] C. Caprini, R. Durrer, and G. Servant, Phys. Rev. D 77, 124015 (2008).

[17] C. Caprini, R. Durrer, T. Konstandin, and G. Servant, Phys. Rev. D 79, 083519 (2009).

[18] L. D. Landau and E. M. Lifshitz, Fluid Mechanics, Course of Theoretical Physics 2nd ed., Vol. 6 (ButterworthHeinemann, London, 1987).

[19] U.-L. Pen and N. Turok, Phys. Rev. Lett. 117, 131301 (2016).

[20] M. Hindmarsh, S. J. Huber, K. Rummukainen, and D. J. Weir, Phys. Rev. D 96, 103520 (2017).

[21] H. Kurki-Suonio and M. Laine, Phys. Rev. D 51, 5431 (1995).

[22] A. H. Guth and E. J. Weinberg, Phys. Rev. D 23, 876 (1981).

[23] K. Enqvist, J. Ignatius, K. Kajantie, and K. Rummukainen, Phys. Rev. D 45, 3415 (1992).

[24] M. Kamionkowski, A. Kosowsky, and M. S. Turner, Phys. Rev. D 49, 2837 (1994).

[25] S. J. Huber and M. Sopena, arXiv:1302.1044.

[26] J. R. Espinosa, T. Konstandin, J. M. No, and G. Servant, J. Cosmol. Astropart. Phys. 06 (2010) 028.

[27] A. Kosowsky and M.S. Turner, Phys. Rev. D 47, 4372 (1993). 